To foster evidence-based forestry approaches among stakeholders globally, the EBF initiative will conduct a “Top 20 Questions for Forestry" (T20Q) project in 2014 Modelled on an earlier exercise that sought to identify key forestry research questions in the United Kingdom and Ireland ${ }^{3}, T 20 Q$ will use an iterative internet survey approach, coupled with workshops and Delphi groups, to determine a set of priority questions in forestry and its associated fields for future systematic reviews, and as a means of identifying potential priorities for further policy development. Through this project, the EBF initiative will foster a 'collaboration without walls' across research and policy organizations concerned with forestry in the contexts of both landscapes and sustainable development.

\section{GETTING INVOLVED}

The EBF initiative was launched in 2013 and is growing collaboratively. You can get involved by engaging with the $T 20 Q$ process, by participating in systematic reviews in your areas of interest and expertise, or by contributing your ideas about progressing the initiative.

Find out more by visiting the EBF website at www.cifor.org/ebf. Contact us at EBFInitiative@cgiar.org with your ideas, or for more information.

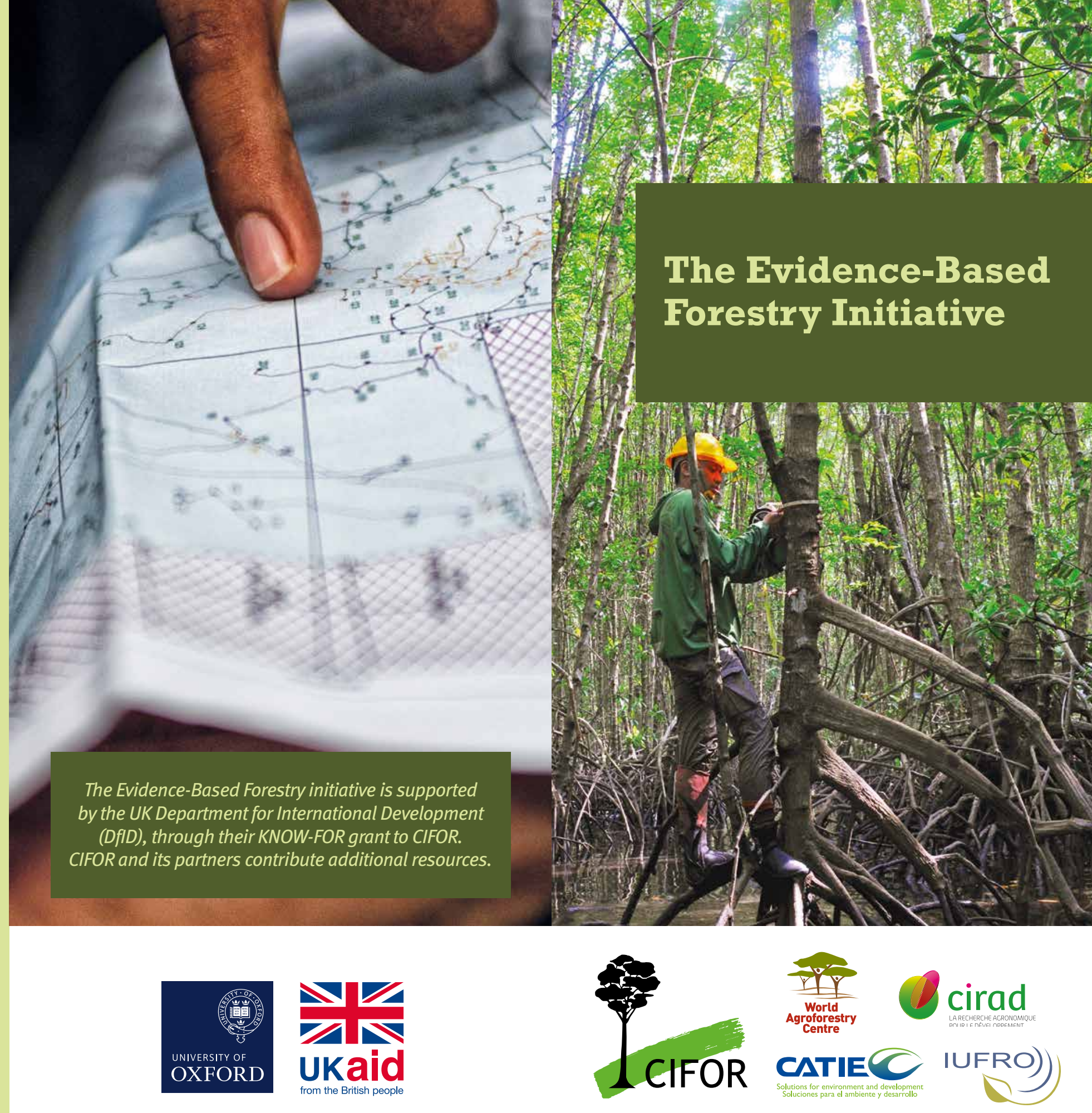


The Center for International Forestry Research (CIFOR) and partner research institutions have key roles in generating evidence for policy processes, both through targeted research and through building the body of relevant knowledge. However, while other sectors have well-established procedures for using systematic approaches to identify and consolidate research findings, transparent methods for assessing a body of evidence have been used only rarely to inform decision-makers in the forestry arena. This opens the potential for bias in the evidence that underpins policy, and for poorlyinformed policy decisions.

Established collaborations in healthcare, social welfare and, most recently, environmental conservation ${ }^{1}$, act as international research networks preparing and disseminating high quality systematic reviews of evidence to contribute to better-informed decisions and better-quality practice Following their lead, CIFOR and its partners established the Evidence-Based Forestry (EBF) initiative, to facilitate use of the best methods for informing decisions and actions in forestry, broadly defined.

The EBF initiative is guided by a Steering Committee comprising representatives from CIFOR, the World Agroforestry Centre (ICRAF), the French Agricultural Research Centre for International Development (CIRAD), the Tropical Agricultural Research and Higher Education Center (CATIE), the International Union of Forest Research Organizations (IUFRO), and the University of Oxford.
As demand for evidence-based approaches continues to grow, the EBF initiative supports and promotes systematic reviews of key policy questions as powerful information tools for decision-making in forestry and related fields. Systematic reviews assess the best available research on a specific question by synthesizing the results of all relevant studies according to carefullydefined and described criteria. This process minimizes bias in presenting the best current evidence, thus facilitating better-informed decision-making.

Systematic reviews begin with the identification of priority questions by stakeholders from research, governmental, and civil society arenas in a collaborative process, thus helping to bridge the persistent divide between research and practice agendas. These selected questions are examined using a rigorous methodology to identify and assess all relevant literature, and the findings are then actively disseminated to inform decision-making in research and policy realms alike.
A systematic review uses transparent procedures to find, evaluate and synthesize the results of relevant research. Procedures are explicitly defined in advance, in order to ensure that the definition of what is relevant is clear, and the reviewing work is transparent and can be replicated (in accordance with normal scientific research practice). This is done both to ensure that reviews can be updated as new relevant information emerges, and to minimize bias.

Studies included in a review are screened for quality, so that the findings of a large number of independent studies can be combined. Peer review is a key part of the process: qualified independent researchers advise on the authors' methods and results, and all stages of the reviews are exposed online for general review by interested parties. The production of a systematic review is a genuinely collaborative effort, as reviews are almost always produced by review teams that bring together methodological and subject expertise.

Steps in the systematic review process from Petrokofsky et al. (2011) ${ }^{2}$

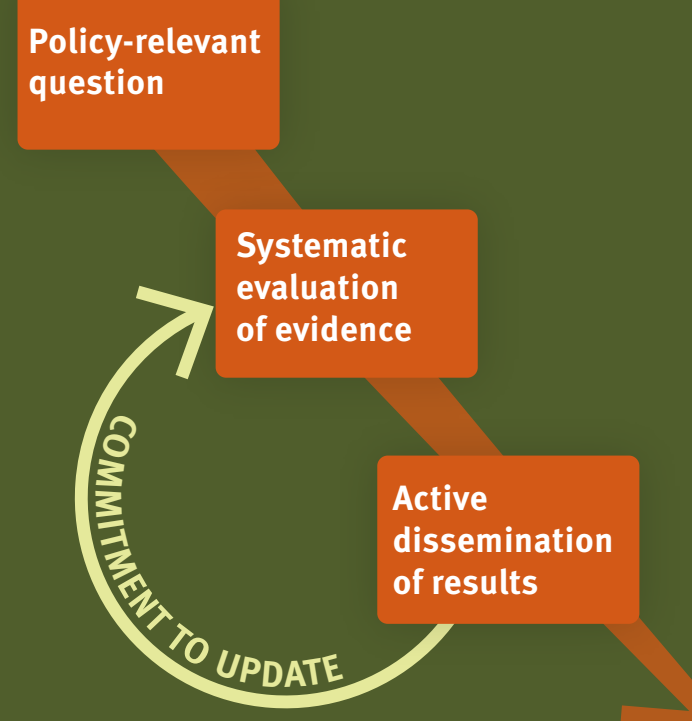

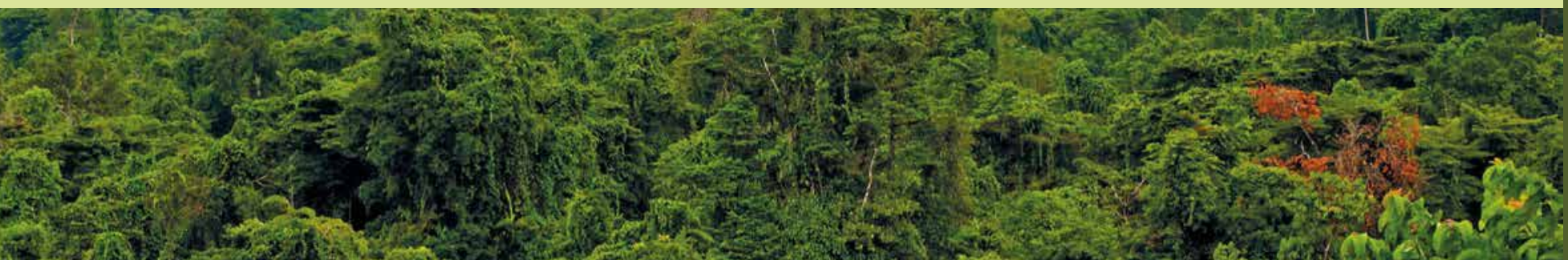

